\title{
Comparison of Serum Folic Acid Level in Oral Lichen Planus Patients and Healthy Subjects
}

\author{
AR Mirzaie ${ }^{1}$, M Hashemi Shahzadeh ${ }^{2}$, M Barzegari ${ }^{3}$, A Azizi ${ }^{4 *}$ \\ ${ }^{I}$ Dentist, member of dental laser research center of Hamedan university \\ ${ }^{2}$ Dentist \\ ${ }^{3}$ Assistant Professor of dermatology, Tehran university of Medical Sciences, Tehran, Iran. \\ ${ }^{4}$ Professor, Oral Medicine Dept,Dental Branch of Tehran, Islamic Azad University, Tehran, Iran.
}

\begin{tabular}{l}
\hline ARTICLE INFO \\
\hline Article Type \\
Original Article \\
\hline Article History \\
Received: Nov 2017 \\
Accepted: Dec 2017 \\
ePublished: Jan 2018
\end{tabular}

Keywords:

Oral Lichen Planus,

Folic Acid,

Antioxidants

\section{ABSTRACT}

Background and aim: Oral lichen planus (OLP) is a chronic mucocutaneous inflammatory disease. Although the etiology of this disease is unknown, some factors including stress, antioxidant deficiency, and folic acid deficiency have been claimed to have a role in its incidence. The purpose of this study was to assess and compare the serum folic acid level in OLP patients and healthy subjects.

Materials and methods: in this case-control study, 52 OLP patients (36 females and 16 males) were selected as the case group, and 48 healthy individuals ( 36 females and 12 males) were assigned to the control group. The serum folic acid level was measured in the two groups. Data were analyzed using T-test with the level of significance set at 0.05 .

Results: the mean and standard deviation (SD) of serum folic acid level was equal to $8.74 \pm 4.11 \mathrm{ng} / \mathrm{ml}$ in the case group and $9.12 \pm 3.5 \mathrm{ng} / \mathrm{ml}$ in the control group with no statistically significant difference $(\mathrm{P}=0.12)$.

Conclusion: The results of the present study indicated that the serum folic acid level in OLP patients did not have a significant difference with that in healthy subjects. 


\section{Introduction:}

Oral lichen planus (OLP) is a chronic mucocutaneous disease with reticular, popular, atrophic/ erosive, plaque-like, and bullous subtypes. ${ }^{(1)}$

Burning sensation and pain are common in atrophic/erosive OLP patients and require treatment. Although the etiology of this disease is unknown, during the recent years, the role of the immune system in its incidence has been confirmed..$^{(2)}$ Recently, other factors including stress, antioxidant deficiency, and the deficiency of micronutrients such as folic acid have been claimed to have a role in the etiology of OLP. ${ }^{(3)}$ Folic acid belongs to the group of $\mathrm{B}$ vitamins and is necessary for cell growth and body metabolism. The word "folate" refers to the folic acid found in natural resources.

Folic acid is vital to a healthy nervous system, blood cells, and stem cells. Folic acid protects the body against heart failure, congenital diseases, cancer, and osteomalacia, and is necessary for cell growth and body metabolism. ${ }^{(4)}$ Moreover, folic acid deficiency causes anemia. The mechanism of action of folic acid is the production of protein in the body and construction of nucleic acids. The complications associated with long-term folic acid deficiency include anemia, redness and swelling of the tongue, diarrhea, psychological disorders such as depression, and reduced growth in children. ${ }^{(5)}$ Researchers have recently focused on the folic acid deficiency in the blood serum of OLP patients. In one study, a folic acid deficiency was observed in OLP patients, ${ }^{(6)}$ while in another study, this condition was detected in $0.3 \%$ of the patients, which was not significant. ${ }^{(7)}$ Considering some shortcomings in this respect and the controversial findings of the previous studies, in this research, we assessed the level of folic acid in OLP patients referring to the dental branch of Islamic Azad University of Tehran and compared it to that in healthy subjects.

\section{Materials and Methods:}

This case-control study was conducted at the oral medicine department of the dental branch of Islamic Azad University of Tehran. 52 OLP patients (36 females and 16 males) were selected as the case group, while 48 healthy individuals (36 females and 12 males) were assigned to the control group.

After taking biopsies from the lesions that clinically resembled OLP lesions, the patients without any signs of dysplasia were included in the case group. The exclusion criteria comprised dysplasia in the histological view, pregnancy, immune disorders, lupus erythematosus, hematologic diseases, systemic diseases, lack of consumption of food supplements such as folic acid by the patients during the last three months, and lichenoid reactions associated with medications or adjacent to dental fillings. ${ }^{(1)}$ This study has been approved by the ethics committee of Islamic Azad University of Tehran (IRCT2014080416090N5). The control group comprised 48 healthy individuals (36 females and 12 males) who had referred to the oral medicine department for periodic examinations and did not have any of the exclusion criteria described for the case group. The participants in the case and control groups were matched according to age and gender. The stages of the study were thoroughly explained to the participants, and 5-ml fasting blood samples were collected from both groups after receiving informed consent forms. To assess the serum folic acid level, the samples were transferred to the laboratory of Razi Hospital, Tehran, Iran, for enzyme immunoassay. This method is based on the competition between serum folate and a labeled folate for a binding protein. This competition between serum folate and the 125I-labeled folate is for binding to the specific protein receptor; therefore, the level of binding of folic acid to the labeled folate is inversely proportional to its concentration in the sample. ${ }^{(5)}$ The data were registered in datasheets and were compared between the two groups using T-test at the significance level of $\mathrm{P}<0.05$ in SPSS 18 software (IBM Co., Chicago, IL, USA). 


\section{Result:}

The mean and standard deviation (SD) of the age was equal to $42.4 \pm 3.8$ years in the case group and $43.5 \pm 2.5$ years in the control group with no statistically significant difference $(\mathrm{P}=0.4)$.

The mean and SD of serum folic acid level was equal to $8.74 \pm 4.11 \mathrm{ng} / \mathrm{ml}$ in the case group and $9.12 \pm 3.5 \mathrm{ng} / \mathrm{ml}$ in the control group with no statistically significant difference $(\mathrm{P}=0.12)$.

There were no statistically significant differences between the two groups in respect to the gender $(\mathrm{P}=0.9)$. Patient information is presented in Table 1.

\section{Table 1: Patient information}

\begin{tabular}{cccc}
\hline Groups & Age (year) & Male & Female \\
\hline Case & $42.4 \pm 3.8$ & 16 & 36 \\
\hline Control & $43.5 \pm 2.5$ & 12 & 36 \\
\hline
\end{tabular}

Reticular mucosal involvement was detected in $44 \%$ of the patients, followed by the erosive subtype (30\%), the popular subtype (16\%), the plaque-like subtype $(8 \%)$, and the bullous subtype (2\%).

\section{Discussion:}

The purpose of the present study was to compare the level of folic acid in OLP patients with that in healthy subjects.

Numerous factors have been considered as the etiology of LP. To date, few studied have been performed on the comparison of serum folic acid in OLP patients and healthy subjects; therefore, the present study is of special value. The results of our study indicated that the serum folic acid level in OLP patients did not significantly differ from that in healthy subjects. This finding is contrary to the results reported by Nosratzehi et al. ${ }^{(6)}$ In an overview study, Nosratzehi showed that folic acid is effective in the treatment of LP. ${ }^{(8)}$ The probable reason for the difference between the results of our study and those of the previous articles can be attributed to the probable differences in the diet of the participants. It can also be attributed to the method of folic acid measurement; for a pre- cise measurement, it is recommended to assess the level of folic acid in red blood cells. In the overview study by Nosratzehi, it has been stated that consumption of folic acid reduces the symptoms of LP; therefore, it may be concluded that vitamins have a therapeutic role rather than an etiologic role. ${ }^{(8)}$ The results of the present study are similar to those reported by Chiang et al as they stated that folic acid deficiency was observed in $0.3 \%$ of the patients and is not a common finding. ${ }^{(7)}$ Also, folic acid deficiency has been proposed as one of the risk factors of depression and psychological stresses. ${ }^{(9)}$ Since stress is one of the factors that can cause LP, ${ }^{(10)}$ folic acid deficiency may be associated with this disease. Although the main cause of LP is unclear, it has been understood that T-cell infiltration (CD4 and particularly CD8) at the epithelium-connective tissue interface is the main cause of this disease. ${ }^{(11)}$

LP is often associated with immunological reactions that microscopically mimic hypersensitivity reactions. Cell-mediated immune responses degenerate the basal layer of the epithelium. The histopathological characteristic of LP comprises the attraction of the lymphocytes to the epithelium-connective tissue interface. ${ }^{(12)}$ Considering the immunologic course of this disease, it seems that folic acid alone does not have a role in the incidence of LP but has a therapeutic role according to the study by Nosratzehi. ${ }^{(8)}$ Folic acid reduces the symptoms of LP through the regulation of the activity of the immune system. ${ }^{(13,14)}$

\section{Conclusion:}

The results of the present study indicated that the serum folic acid level in OLP patients did not have a significant difference with that in healthy subjects.

Aknowledgement:

This article is based on General Dentistry Thesis ,No: 11087. Also, we are thankful to Mr. Naser Valaei regarding to his cooperation in preparing of statistical analysis.

\section{References:}

1- Jones KB, Jordan R. White lesions in the oral cavity: clinical presentation, diagnosis, and treatment. Semin Cutan Med Surg. 2015 Dec;34(4):161-70. 
2- Lu R, Zhang J, Sun W, Du G, Zhou G. Inflammation-related cytokines in oral lichen planus: an overview. J Oral Pathol Med. 2015 Jan;44(1):1-14. 3- Azizi A, Farshchi F. Comparison of salivary and plasma antioxidant levels in lichen planus patients and healthy subjects. J Oral Pathol Med. 2012 Aug;41(7):524-6.

4- Morris MS, Jacques PF, Rosenberg IH, Selhub J. Folate and vitamin B-12 status in relation to anemia, macrocytosis, and cognitive impairment in older Americans in the age of folic acid fortification. Am J Clin Nutr. 2007 Jan;85(1):193-200.

5- Chang JY, Chen IC, Wang YP, Wu YH, Chen HM, Sun A. Anemia and hematinic deficiencies in gastric parietal cell antibody-positive and antibodynegative erosive oral lichen planus patients with thyroid antibody positivity. J Formos Med Assoc. 2016 Nov;115(11):1004-11.

6- Nosratzehi T, Ansari-Moghaddam A, Arbabi-Kalati F, Maleki L, Amiriyan A. Effect of the serum vitamin B12 and folic acid levels in oral lichen planus. J Res Dent Sci. 2013;9(4):187-91.

7- Chiang CP, Yu-Fong Chang J, Wang YP, Wu YH, Lu SY, Sun A. Oral lichen planus - Differential diagnoses, serum autoantibodies, hematinic deficiencies, and management. J Formos Med Assoc. 2018 Feb 19. pii: S0929-6646(18)30052-4.

8- Nosratzehi T. Oral lichen planus: an overview of potential risk factors, biomarkers and treatments. Asian Pac J Cancer Prev. 2018 May 26;19(5):116167.

9- Kemse NG, Kale AA, Joshi SR. A combined supplementation of omega- 3 fatty acids and micronutrients (folic acid, vitamin B12) reduces oxidative stress markers in a rat model of pregnancy induced hypertension. PloS One. 2014 Nov18;9(11):e111902.

10- Kalkur C, Sattur AP, Guttal KS. Role of depression, anxiety and stress in patients with oral lichen planus: a pilot study. Indian J Dermatol. 2015 SepOct;60(5):445-9.

11- Azizi A, Lawaf S. The comparison of efficacy of adcortyl ointment and topical tacrolimus in treatment of erosive oral lichen planus. J Dent Res Dent Clin Dent Prospects. 2007 Fall;1(3):99-102.

12-Vered M, Furth E, Shaley Y, Dayan D. Inflammatory cells of immunosuppressive phenotypes in oral lichen planus have a proinflammatory pattern of expression and are associated with clinical parameters. Clin Oral Investig. 2013 Jun;17(5):1365-73.

13- Li S, Zhi L, Liu Y, Shen J, Liu L, Yao J, et al. Effect of in ovo feeding of folic acid on the folate metabolism, immune function and epigenetic modification of immune effector molecules of broiler. $\mathrm{Br}$ J Nutr. 2016 Feb 14;115(3):411-21.
14- Patearroyo T, Ubeda N, Montero A, Achon M, Varela-Moreiras G. Vitamin B(12) and folic acid imbalance modifies NK cytotoxicity, lymphocytes B and lymphoprolipheration in aged rats. Nutrients. 2013 Nov 26;5(12):4836-48. 